\title{
Ever intriguing 'Primary Amenorrhea'- an audit
}

\section{Sapna Vinit Amin*, Lavanya Rai, Priyadharshini Palpandi, Aswathy Kumaran}

Department of Obstetrics \& Gynecology, Kasturba Medical College, Manipal University, Manipal, Karnataka, India

Received: 30 October 2014, Revised: 15 November 2014

Accepted: 16 November 2014

\section{*Correspondence:}

Dr. Sapna Vinit Amin,

E-mail: astra117us@gmail.com,drsapnagoni@rediffmail.com

Copyright: () the author(s), publisher and licensee Medip Academy. This is an open-access article distributed under the terms of the Creative Commons Attribution Non-Commercial License, which permits unrestricted non-commercial use, distribution, and reproduction in any medium, provided the original work is properly cited.

\begin{abstract}
Background: Primary amenorrhea though its incidence is less than $0.1 \%$ often is a diagnostic challenge in establishing etiology and causes management dilemma to the gynecologist in the developing countries.

Methods: A retrospective audit of all the cases of primary amenorrhea coming to our tertiary care teaching hospital in South India over 6 years, from January 2006 to December 2012.

Results: Ninety-eight cases of primary amenorrhea were analyzed and it was found that the causes of primary amenorrhea were structural anomalies in $47.9 \%$, endocrinological abnormality in $31.6 \%$, and genetic factors in $20.5 \%$ of the patients. Mullerian anomalies were the most common cause for primary amenorrhea compared to gonadal dysgenesis in our study group; hence surgical correction formed an important mode of treatment following diagnosis.

Conclusions: Primary amenorrhea work-up may seem to be complex, nevertheless a well elicited history, carefully conducted physical examination followed by use of imaging modalities and bio assays for endocrine abnormalities, permitted the clinician to narrow the diagnostic possibilities and reach an accurate diagnosis quickly that helped in choosing the appropriate management option. While managing, counselling of the patient and her near relatives was very essential for fruitful outcome. Among the surgical approaches for management, sigmoid vaginoplasty was found to be a promising alternative to MacIndoe's vaginoplasty in our series.
\end{abstract}

Keywords: Primary amenorrhea, Mullerian anomalies, Gonadal dysgenesis, Sigmoid vaginoplasty

\section{INTRODUCTION}

Amenorrhea refers to the absence of menstrual cycles. It may be classified as primary, if menstrual bleeding has never occurred in the absence of hormonal treatment. An evaluation of primary amenorrhea must be initiated in the absence of menses by the age 15 regardless of presence of normal growth and development of secondary sexual characters or by the age of 13 in the absence of growth or development of secondary sexual characters. ${ }^{1,2}$

Primary amenorrhea though uncommon (incidence less than $0.1 \%$ ) often is a diagnostic challenge in establishing aetiology. Choosing the appropriate line for managing it poses a dilemma to the gynecologist in the developing countries. This is because the aetiopathology for primary amenorrhoea may well be one that affects the girl's fertility, sexuality and reproductive function. In a developing country like India, this is associated with social stigma. Hence unless a holistic management approach is adopted more harm than good may be achieved.

Most of the literature published on primary amenorrhoea often analysed the aetiopathology and stressed less on the evaluation and management aspects. ${ }^{3-6}$ Therefore through this audit we aim to empower the medical practitioners in developing countries to effectively evaluate a patient with primary amenorrhoea and help determine the best management line and implement it.

\section{METHODS}

A retrospective audit of all the cases of primary amenorrhea coming to our tertiary care teaching hospital in South India over 6 years, from January 2006 to 
December 2012 was conducted. Primary amenorrhoea was defined as absence of menstruation by the age 15 regardless of presence of normal growth and development of secondary sexual characters or by the age of 13 in the absence of growth or development of secondary sexual characters.

The data was obtained from the case records of all patients who presented to our centre satisfying the abovementioned criteria for primary amenorrhoea. Ninety-eight cases of primary amenorrhea were analyzed. The epidemiological details, presenting complaints, clinical evaluation, investigative findings and management details of each patient were collected and critically analysed. The collected data was analysed using Microsoft excel and incidences and frequencies estimated.

\section{RESULTS}

Ninety-eight patients who presented with primary amenorrhoea as defined earlier were included in the audit. The age of the patients at presentation ranged between 13- 40 years (average age- 26.5 years).

\section{The presenting complaints}

Among the 98 patients analysed, 79 presented with amenorrhea, 19 patients presented with cyclical abdominal pain, eight with primary infertility. Weight loss was noted in three, one patient had multiple small joint pains and another one patient was a follow up case of treated ovarian germ cell tumor. Two patients had coexisting type 1 diabetes mellitus and one had headache and visual disturbances. Sixty-one patients had normal secondary sexual characters, 34 had absent secondary sexual characters and three had virilising features.

\section{The diagnoses (Table 1, Figure 1)}

Definitive aetiology for primary amenorrhoea could be established in $91(92.8 \%)$ patients out of 98. Only in seven $(0.07 \%)$ girls, constitutional delay was determined as the cause for primary amenorrhoea as a diagnosis of exclusion. Following thorough clinical and investigative evaluation, $47(47.9 \%)$ patients were diagnosed to have anatomical defects, $31(31.6 \%)$ had endocrinological abnormalities and $20(20.5 \%)$ had genetic abnormality. Amongst those with anatomical defects, 34 (78.7\%) had Mullerian agenesis or Meyer-Rokitansky-Kustner-Hauser syndrome, one had imperforate hymen, 12 had cervicovaginal/vaginal atresia. All these women had normal female genotype.

Gonadal dysgenesis/genetic causes accounted for amenorrhoea in 20 patients. Karyotyping revealed that 12 $(60 \%)$ women had gonadal dysgenesis due to Turners syndrome $(45, \mathrm{XO})$. Two patients had pure gonadal dysgenesis, in whom the normal female karyotype (46, $\mathrm{XX)}$ was seen. Five patients' karyotyping revealed presence of Y chromosome, three of whom had androgen insensitivity, and two, 5-alpha reductase deficiency rendering them phenotypically female. One patient had mixed gonadal dysgenesis with karyotype showing $\mathrm{XO} / \mathrm{XY}$ mosaic.

Endocrine dysfunction/immaturity accounted for amenorrhoea in 31 patients. Premature ovarian insufficiency was the cause in 11 patients while eight had central pathology (hypogonadotrophic hypogonadism) girls had. Only seven girls were found to have constitutional delay in achieving menarche with no other obvious pathology. Two girls had congenital adrenal hyperplasia and two others had severe hypothyroidism accounting for their amenorrhoea.

Table 1: Specific causes for primary amenorrhea.

\begin{tabular}{|ll|}
\hline \multicolumn{2}{|l|}{ Specific causes for primary amenorrhea } \\
\hline Structural causes (n=47) \\
\hline Mullerian agenesis/hypoplasia & 34 \\
\hline Imperforate hymen & 1 \\
\hline Vaginal agenesis & 8 \\
\hline Cervico vaginal atresia & 4 \\
\hline Endocrinological causes (n=31) & \\
\hline Hypogonadotropic hypogonadism & 9 \\
\hline Primary ovarian failure & 11 \\
\hline Congenital adrenal hyperplasia & 2 \\
\hline Constitutional delay & 7 \\
\hline Hypothyroidism & 2 \\
\hline Genetic causes (n=20) & \\
\hline Turner syndrome & 12 \\
\hline Pure gonadal agenesis & 2 \\
\hline Androgen insensitivity syndrome & 3 \\
\hline Mixed gonadal dysgenesis & 1 \\
\hline 5-alpha reductase deficiency & 2 \\
\hline
\end{tabular}

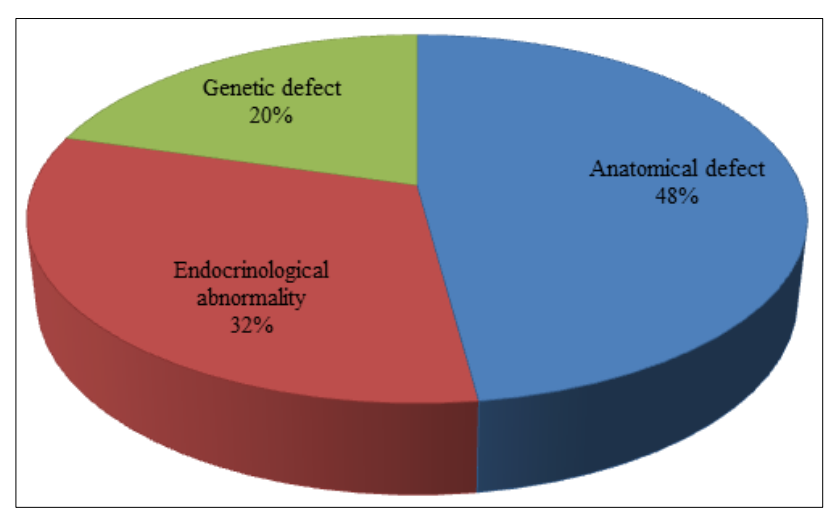

Figure 1: Aetiology of primary amenorrhea.

\section{Management}

\section{Genital tract anomalies (Figure 2)}

Imperforate hymen was treated surgically in one patient. Transverse vaginal septum excision was done in another. Eight patients were treated with vaginoplasty. Sigmoid 
vaginoplasty was done in five cases, while three girls underwent Mc Indoe vaginoplasty. Six patients with narrow short vagina were managed successfully with serial vaginal dilatation. The patients who underwent corrective surgical procedures/dilatation included women with gonadal dysgenesis/chromosomal anomalies also.

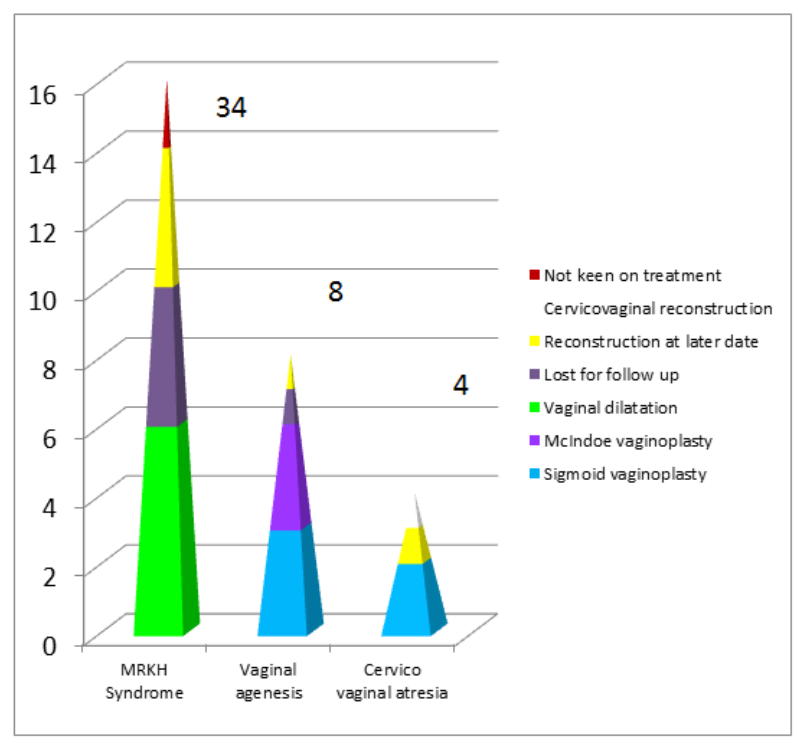

Figure 2: Surgeries done for Mullerian anomalies.

\section{Genetic causes (Figure 3)}

All the 12 cases of Turner syndrome received hormone replacement therapy. Amongst these, six patients had withdrawal bleeding, three had improved secondary sexual characters, one had been just started on treatment, and two were lost for follow up. Of the three patients with androgen insensitivity syndrome two patients had orchidectomy and were started on hormone replacement, the third patient was advised gonadectomy, but lost for follow up. One patient among the two, with 5-alpha reductase deficiency underwent gonadectomy, sigmoid vaginoplasty, and was started on hormone replacement therapy, but the other patient was lost for follow up.

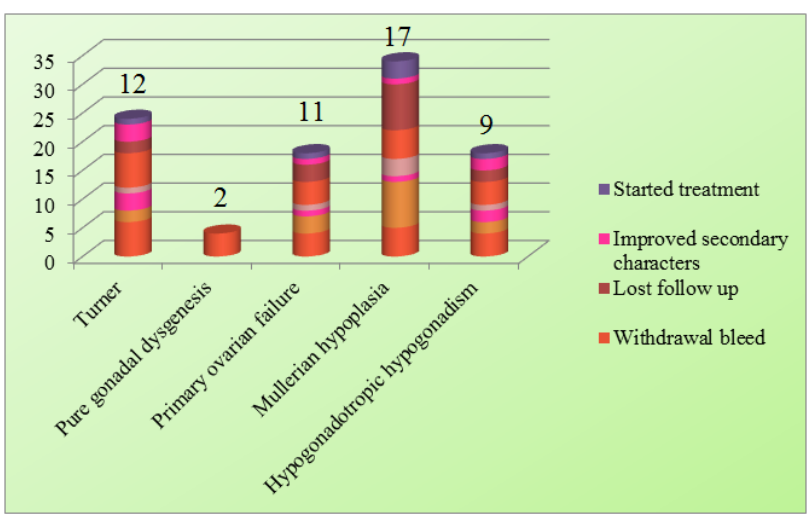

Figure 3: Treatment and follow up of patients.

\section{Endocrine abnormalities}

The seven girls with constitutional delay were reassured and all of them achieved menarche spontaneously within one year. Two patients with congenital adrenal hyperplasia were treated with steroids and underwent vaginal dilatation. The two girls with hypothyroidism had favourable outcomes following thyroxine replacement therapy.

The girls with primary ovarian failure and normal karyotype were evaluated for the cause for same and child hood tuberculosis was found to be the culprit in three girls. One girl had developed ovarian failure following early childhood treatment for ovarian germ cell tumour. Severe insulin dependent diabetes was the pathology one patient but no definitive aetiology could be established for the other seven patients. These patients were started on cyclical hormone replacement therapy to ensure menstruation as well as for osteoprotection.

The girls with central causes for amenorrhoea were managed in conjunction with the neurologists.

\section{DISCUSSION}

Primary amenorrhea though uncommon results in significant anguish to the patient and her relatives and hence establishing the accurate aetiology and optimum management are of paramount importance. This audit conducted in our South Indian tertiary care hospital aimed at using this important tool of systematic clinical review for optimising patient care and management.

\section{Presentation}

Published literature from western countries often mention primary amenorrhea patients presenting with complaints of short stature rather than amenorrhea, where most often the pathology is hypogonadism rather than anatomical defects. ${ }^{1,2}$ Unlike western reports maximum patient population in our study presented with amenorrhea. This observation may be because the most of patients and their relatives in our set up were more concerned about attaining menarche that would establish their reproductive maturity and also because Mullerian anomalies were the most common cause of amenorrhea in our population subset.

Ashok Krishna et al. who did a study similar to ours in Assam, North West India also noted that amenorrhoea was the most frequent presenting complaint. ${ }^{3}$ They also noted that the average age of presentation was $17.23 \pm$ 4.2 years. $^{3}$ In our centre the average age of presentation was around 26.5 years, the reason being that the patients were incompletely evaluated and managed at various peripheral hospitals before being referred. The average age of initial presentation with amenorrhea was 16.8 years that was comparable to that noted by Ashok Krishna et al. 


\section{Aetiology}

Gynecology textbooks often mention gonadal dysgenesis as the major etiological factor accounting for $30-40 \%$ cases of primary amenorrhea, which often present with the associated physical features like short stature. Mullerian anomalies are often cited as an uncommon cause for primary amenorrhea. ${ }^{1,2}$

This audit we conducted in the South Indian scenario revealed that genital tract developmental abnormalities without gonadal dysfunction was responsible for primary amenorrhea in $47.9 \%$ while gonadal dysgenesis/genetic abnormalities accounted only for $20.5 \%$. These findings were different from that cited in western textbooks. Interestingly, our observations were similar to a study conducted in Thailand by Tanmahasamut $\mathrm{P}$ et al., where they analyzed 295 cases of primary amenorrhea and found that the most common cause was Mullerian agenesis (39.7\%), followed by gonadal dysgenesis $(35.3 \%){ }^{4}$ A Srilankan study published by Samarakoon et al. also showed incidence of gonandal dysgenesis as $20.6 \%$ in patients with primary amenorrhoea. ${ }^{5}$ Hence, racial and environmental factors may have role in the aetiology of primary amenorrhea.

Endocrine abnormalities were the second most frequent cause for primary amenorrhea among our patients.

Gonadal dysgenesis/chromosomal anomalies accounted for only $20.5 \%$ as mentioned earlier. Gonadal dysgenesis/chromosomal abnormalities were suspected in patients in whom on thorough physical and radiological evaluation were found to have streak/ectopic gonads. In concordance with literature, subsequent karyotyping revealed Turners syndrome as the most frequent cause for gonadal dysgenesis $(60 \%)$. Five patients had genotype 46, XY three of whom had androgen insensitivity syndrome and two, 5-alpha reductase deficiency. Two girls have pure gonadal dysgenesis with karyotype 46, $\mathrm{XX}$ and one patient had mixed gonadal dysgenesis. As gonadectomy is advised in dysgenetic/ectopic gonads harbouring Y chromosome, the karyotyping formed a very important diagnostic tool.

\section{Evaluation and management}

Through our audit we were able to find that a comprehensive clinical history followed by thorough physical examination in conjunction with a simple diagnostic abdomino-pelvic ultrasound were all that was required to form a provisional diagnosis for the cause for primary amenorrhea. We would like to stress that examination under anaesthesia formed an invaluable part on complete physical examination where mandated. As most of the patients were young/unmarried, empathetic counselling of the patient and her relatives was done so that the evaluation and management could proceed without impediment.
A routine hemogram, blood sugar evaluation, thyroid function tests and serum prolactin levels were also part of primary amenorrhea evaluation. The chance of pregnancy was ruled out in all the women. The presence of a withdrawal bleed helped in ruling out an endometrial and outflow tract pathology. In the absence of outflow tract abnormality thin unresponsive endometrium should lead to suspicion of Ashermans/genito urinary Kochs.

The physical evaluation and clinical history helped in categorising whether the amenorrhoea was due to anatomical abnormalities, gonadal/chromosomal abnormalities, endocrinopathies or central/hypothalamopituitary origin. Here we would like to mention that the management of primary amenorrhea often involved a multidisciplinary team that included the paediatrician, the neurologist, the endocrinologist, the genito urinary surgeon as well as the clinical psychologist along with the gynaecologist.

MRI scan was used as the gold standard for evaluating Mullerian and outflow tract as well as coexisting genitourinary anatomical abnormalities. Obstructive pathology like imperforate hymen and transverse vaginal septae was easily tackled by the experienced gynaecologist with the patients subsequently attaining normal menstrual and reproductive capacity.

More severe pathology like vaginal agenesis, cervical agenesis or other coexisting correctable genitourinary anomalies was challenging. The patient and relatives had to be convinced regarding the reconstructive surgery if possible as well as counselled about the long term after care following same. Such surgeries required the expertise of a pelvic reconstructive surgeon, urologist and paediatric surgeon and prognosis depended on surgical success as well as patient compliance.

The patients with Mullerian agenesis were counselled that they would be able to have satisfactory sexual relationship following vaginoplasty/vaginal dilatation though they would not menstruate due to the absence of uterus. They were also told that as they have healthy functional ovaries they might try for biological offspring following IVF and embryo-tranfer into a surrogate.

Patients with suspected hypogonadism were further evaluated to determine whether it was due to central pathology or not. High FSH and LH in the presence of unresponsive ovaries were suggestive of ovarian pathology and all of those patients underwent karyotyping. The patients with normal karyotype were evaluated for other causes of premature ovarian insufficiency like autoimmune diseases, history of infective/iatrogenic/traumatic damage to gonads, IDDM etcetera.

The patients with male karyotype were counselled for gonadectomy, followed by vaginoplasty/dilatation as was required and hormone replacement therapy. They and their relatives were counselled about the patient's 
inability to menstruate or conceive though she can have a satisfactory conjugal relationship.

The patients with Turners syndrome, pure gonadal dysgenesis and primary ovarian insufficiency were started on hormone replacement treatment along with the management of coexisting medical pathology if any. They were also made aware of the fact that though they might menstruate and their uterus could be capable of carrying a pregnancy, their ovaries were non-functional and they could not have their own biological offspring. These patients were counselled that they had the option for donor egg IVF if they wanted to carry their partners' biological offspring later.

Patients, in whom evaluation revealed hypothyroidism, were started on treatment in conjunction with the endocrinologist. Other endocrine pathology like congenital adrenal hyperplasia, hyperprolactinaemia, Cushing's syndrome etcetera presented with characteristic clinical findings and were confirmed by the respective diagnostic hormone tests. Polycystic ovarian syndrome though is increasingly being attributed as a cause of primary amenorrhea, was not seen in the patients in our study. Patients with treatable endocrine abnormalities and functional ovaries were reassured that they will attain menarche and have abnormal reproductive life with appropriate treatment.

Women with features suggestive of hypothalomopituitary hypo function/tumours were evaluated alongside the neurologist and managed based on the respective causative aetiology. Menstrual and reproductive prognosis were explained based on those. Those with hypogonadotropic hypogonadism were counselled about the ever expanding frontiers of ART open to them with which they could have their own biological offspring.

\section{Vaginoplasty}

Since anatomical abnormalities, especially Mullerian agenesis as well as gonadal dysgenesis with rudimentary vagina, were frequent in our patient subset, vaginoplasty formed an important therapeutic component in these patients.

In our setup we found sigmoid vaginoplasty to be advantageous over the traditional McIndoe vaginoplasty, as it provided good vaginal length, adequate secretion allowing lubrication, less incidence of vaginal stenosis, it also did not need prolonged neovaginal dilatation and more importantly had short recovery time with good patient satisfaction. The patient follow up over 5 years showed good functional outcome. In sigmoid vaginoplasty, a section of the recto sigmoid colon was transplanted into a newly created vaginal tunnel forming a neovaginal (Figure 4). Nowier et al. and Kapoor et al. have cited satisfactory long outcomes following sigmoid vaginoplasty. ${ }^{8,9}$ In the recent decades novel vaginoplasty techniques like Vecchietti and Davydov procedures have become more popular and practiced in many of the
European countries (both open and laparoscopic techniques. ${ }^{10}$

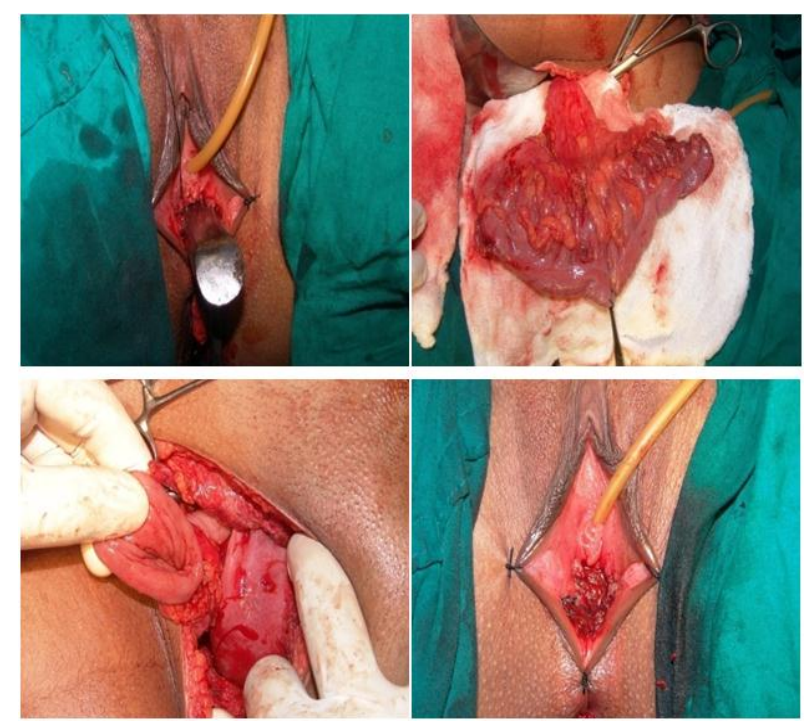

Figure 4: Sigmoid vaginoplasty.

\section{Psychosexual counselling}

Primary amenorrhea and the its causative pathology often affected the psyche of the patient and her family. The younger the patient the greater her emotional immaturity, hence greater was the need for formative counselling for her and family so that favourable overall outcomes were achieved. ${ }^{2,11}$

Because of the sexual nature of a reproductive disorder, the social stigma associated made it difficult for families. Families were educated regarding their ward's condition and what her sexual and reproductive capacity was. The parents were helped so that they could come to terms with their own feelings first regarding the diagnosis and prognosis so that they could help and support their child.

They were encouraged to be honest when they plan her marriage so that, marital discord that could arise from the spouse's unawareness about patient's condition might be avoided .The intention of counselling was to help the young patient formulate positive self-esteem and body image, despite impaired fertility if present. ${ }^{1,2,11}$

\section{Follow up}

All the patients and their care providers had been counselled about the importance of follow up visits for reassessment and modification as might be required according to.

In our study group, the overall follow up rate was $86 \%$ at the end of one year and it dropped to $50 \%$ at the end of 3 years. Most of the dropouts were those with minor pathology and good reproductive outcomes who had achieved menarche. Twenty percent of those with MRKH syndrome and androgen insensitivity opted to follow up 
corrective surgery.

Table 2: Comparison with other studies.

\begin{tabular}{|c|c|c|c|c|c|}
\hline & $\begin{array}{l}\text { Present study } \\
\text { (2012) }\end{array}$ & $\begin{array}{l}\text { Ashok Krishna } \\
\text { et al. (2012) }\end{array}$ & $\begin{array}{l}\text { Tanmahasamut P } \\
\text { et al. (2012) }\end{array}$ & $\begin{array}{l}\text { Samarakoon } \\
\text { et al. (2013) }\end{array}$ & $\begin{array}{l}\text { Faeza et al. } \\
(2012)\end{array}$ \\
\hline Population studied & South India & North India & Thailand & Srilanka & Egypt \\
\hline Mean age of presentations & 26.5 years & 17.23 years & - & - & - \\
\hline Chief complaints & $\begin{array}{l}\text { Primary } \\
\text { amenorrhoea }\end{array}$ & $\begin{array}{l}\text { Primary } \\
\text { amenorrhoea }\end{array}$ & - & - & - \\
\hline Commonest Cause & $\begin{array}{l}\text { Mullerian } \\
\text { anomalies }\end{array}$ & $\begin{array}{l}\text { Mullerian } \\
\text { anomalies }\end{array}$ & $\begin{array}{l}\text { Mullerian anomalies } \\
(39.7 \%)\end{array}$ & - & - \\
\hline $\begin{array}{l}\text { Frequency of chromosomal } \\
\text { anomalies }\end{array}$ & $21.00 \%$ & - & $35.3 \%$ & $20.63 \%$ & - \\
\hline
\end{tabular}

\section{CONCLUSIONS}

This thorough audit has helped us in formulating a more streamlined and efficient evaluation and management plan for primary amenorrhoea in the South Indian scenario. A well-elicited clinical history, carefully conducted physical examination followed by use of imaging modalities and bioassays for endocrine abnormalities were the keys to unlocking the aetiology.

Mullerian agenesis and developmental genital tract abnormalities were the more frequently (47.9\%) accounted for primary amenorrhea compared to gonadal dysgenesis $(20.5 \%)$ in our population subset. Consequently, corrective surgery formed an important part of management. Among the surgical approaches for management, sigmoid vaginoplasty was seen to be a better alternative to MacIndoe's vaginoplasty among our patients.

When managing primary amenorrhea, in depth counseling of the patient and her relatives; so that they were made aware of what was wrong, what they should expect and what was required of them, was found to be very essential for fruitful therapeutic outcome.

\section{ACKNOWLEDGEMENTS}

We would like to acknowledge professors Dr. Pratap Kumar and Dr. Muralidhar V Pai and Additional professor Dr Jyothi Shetty, department of obstetrics \& gynecology, Kasturba medical college, Manipal University, Manipal for providing generous academic insight for our study. In addition, we would like to acknowledge the faculty from pediatric surgery department, Kasturba medical college, Manipal University, Manipal for valuable guidance.

Funding: No funding sources

Conflict of interest: The authors of this publication, are affiliated to department of obstetrics \& gynecology, Kasturba Medical College, Manipal University, Manipal. The terms of this arrangement have been reviewed and approved by the Manipal University, Manipal, in accordance with its policy on objectivity in research.

Ethical approval: The study was approved by the Manipal University Ethics Committee

\section{REFERENCES}

1. Jonathan S. Berek. Puberty. In: Jonathan S. Berek, eds. Berek and Novaks Gynecology. 15th ed. Philadelphia: Lippincott Williams and Wilkins; 2011: 991-1034.

2. Fritz MA, Speroff L. Amenorrhoea. In: Fritz MA, Speroff L, eds. Clinical Gynaecologic Endocrinology and Infertility. 8th ed. New Delhi: Wolters Kluwer, Lippincott Williams \& Wilkins; 2011: 1352-1354.

3. Ashok Krishna Bhuyan, Dipti Sarma, Uma Kaimal Saikia. Contemporary issues in primary amenorrhea: an experience from a tertiary care centre. Indian $\mathbf{J}$ Endocrinol Metab. 2012;16(2):387-8.

4. Tanmahasamut P, Rattanachaiyanont M, Dangrat C, Indhavivadhana S, Angsuwattana S, Techatraisak K. Causes of primary amenorrhoea: a report of 295 cases in Thailand. J Obstet Gynecol Res. 2012;1:297-301.

5. Samarakoon L, Sirisena ND, Wettasinghe KT, Kariyawasam KW, Jayasekara RW, Dissanayake VH. Prevalence of chromosomal abnormalities in Sri Lankan women with primary amenorrhoea. J. Obstet Gynecol Res. 2013;39(5):991-7.

6. Faeza El- Dahtory. Chromosomal abnormalities and hormonal disorders of primary amenorrhea patients in Egypt. Indian J Hum Genet. 2012;18(2):183-6.

7. Welt CK. Primary ovarian insufficiency: a more accurate term for premature ovarian failure. Clin Endocrinol (Oxf). 2008;68(4):499-509.

8. Kapoor R, Sharma DK, Singh KJ, Suri A, Singh P, Chaudhary H, et al. Sigmoid vaginoplasty: long-term results. Urology. 2006 Jun;67(6):1212-5.

9. Nowier A, Esmat M, Hamza RT. Surgical and functional outcomes of sigmoid vaginoplasty among 
patients with variants of disorders of sex development. Int Braz J Urol. 2012;38(3):380-6.

10. Ismail IS, Cutner AS, Creighton SM. Laparoscopic vaginoplasty: alternative techniques in vaginal reconstruction. BJOG. 2006;113:340-3.
11. Iglesias EA, Coupey SM. Menstrual cycle abnormalities: diagnosis and management. Adolesc Med. 1999;10(2):255-73.

DOI: $10.5455 / 2320-1770 . i j r c o g 20141242$

Cite this article as: Amin SV, Rai L, Palpandi P, Kumaran A. Ever intriguing 'Primary Amenorrhea'an audit. Int J Reprod Contracept Obstet Gynecol 2014;3:1090-6. 